\title{
PREVALÊNCIA DE SOBREPESO E OBESIDADE EM CRIANÇAS E ADOLESCENTES DA CIDADE DE MACEIÓ
}

\author{
Maria Roseane Tenorio Mendonça ${ }^{1 *}$, Maria Alayde Mendonça da Silva², Ivan Romero Rivera ${ }^{3}$, Adriana Ávila Moura ${ }^{4}$ \\ Trabalho realizado no departamento de Tocoginecologia e Pediatria do Hospital Universitário Prof. Alberto Antunes - Universidade Federal de Alagoas - UFAL, \\ Maceió, AL
}

\author{
*Correspondência: \\ Rua: Ind. José Otávio \\ Moreira, 73 Apto-201, \\ Jatiúca \\ Maceió-AL \\ CEP: $57036-600$ \\ Tel: (82) 3235-2158; \\ 3325-2905, 9974-4233 \\ eqsaude@hotmail.com.br
}

\begin{abstract}
RESUMO
OвJETIvo. Estabelecer a prevalência de sobrepeso e obesidade em crianças e adolescentes de Maceió (Alagoas, Brasil) e verificar associação destes fatores com gênero, idade e tipo de escola.

Métodos. Estudo epidemiológico descritivo, transversal. Avaliou jovens de 7 a 17 anos de idade, sorteados das 396 escolas públicas e particulares de Maceió. Após a randomização, os dados foram coletados por meio de questionário e medidas antropométricas (Índice de Massa Corporal = peso: altura ${ }^{2}$ ). Sobrepeso e obesidade foram definidos como Índice de Massa Corporal superior ao percentil 85 e igual ou superior ao percentil 95 para idade e sexo, respectivamente.

Resultados. A amostra final foi constituída de 1253 estudantes (706 do gênero feminino). Identificados 116 estudantes com sobrepeso e 56 estudantes com obesidade. Houve associação significante entre obesidade e estudantes com idade de sete a nove anos quando comparados com os de 10 a 13 anos $(p<0,04)$ e com os de 14 a 17 anos $(p<0,02)$. Estudantes das escolas particulares apresentaram 0 dobro de chance de terem sobrepeso ( $\mathrm{OR}=2,2$; IC95\%:1,36-3,32) e aproximadamente cinco vezes mais chances de apresentarem obesidade $(\mathrm{OR}=4,7$; IC95\%:2,32-9,34) em relação aos das escolas públicas.
\end{abstract}

ConcLusão. As prevalências do sobrepeso e obesidade foram de 9,3\% e 4,5\% respectivamente, sendo significantemente mais elevadas em estudantes das escolas particulares.

Unitermos: Prevalência. Sobrepeso. Obesidade. Criança. Adolescente. Antropometria.

\section{INTRODUÇÃO}

A obesidade é uma doença crônica em expansão e sua prevalência vem aumentando de forma crescente em todas as faixas etárias, em todos os estratos sociais, tanto em países desenvolvidos quanto em desenvolvimento, o que a torna epidêmica em alguns países ${ }^{1-3}$.

Estudos epidemiológicos de base populacional que investigam sobrepeso e obesidade na infância são necessários na atualidade. A prevalência de sobrepeso em crianças americanas de 6 a 11 anos é de 13,7\%. Em crianças inglesas, o sobrepeso é de $25 \%$ entre aquelas de 5 a 11 anos. No Brasil, pesquisas nacionais evidenciaram que $4,8 \%$ das crianças menores de 10 anos e $4,6 \%$ entre um a quatro anos têm sobrepeso $0^{4-7}$.

No Brasil, a prevalência de desnutrição em adultos e crianças tem apresentado redução importante, enquanto a obesidade vem se elevando ao longo do tempo. Estudos nacionais representativos, de 1974 a 1989, com adultos e crianças, demonstram que o país vem apresentando rápida modificação, reduzindo os problemas quanto ao déficit da oferta alimentar e passando para as repercussões patológicas do excesso dessa oferta na população, constituindo a chamada transição nutricional ${ }^{7}$.

Inquéritos nacionais realizados nas décadas de 70, 80 e 90, no Brasil, demonstraram crescimento na prevalência de obesidade de 3,6\% para 7,6\% no gênero feminino, na população de quatro a cinco anos de idade. Escola privada (classe média / alta) na região Nordeste apresentou prevalências de $26,2 \%$ de sobrepeso e $8,5 \%$ de obesidade em crianças e adolescentes. Entretanto, não há informações representativas sobre o perfil nutricional da população, em qualquer faixa etária, no Estado de Alagoas ou em seus municípios ${ }^{7-8}$.

0 presente estudo teve como objetivos estabelecer a prevalência do sobrepeso e obesidade em crianças e adolescentes da cidade de Maceió, além de verificar associações dessas variáveis com gênero, idade e tipo de escola.

\section{MÉtodos}

Estudo epidemiológico, descritivo, observacional, de corte transversal e base populacional, realizado em amostra

1. Mestre - professora do Instituto Batista de Ensino Superior de Alagoas - IBESA, Maceió, AL

2. Doutora - professora adjunta do departamento de Clínica Médica da Universidade Federal de Alagoas - UFAL, Maceió, AL

3. Doutor - professor adjunto do departamento de Tocoginecologia e Pediatria da Universidade Federal de Alagoas - UFAL, Maceió, AL

4. Mestre - professora do departamento de Tocoginecologia e Pediatria - da Universidade Federal de Alagoas - UFAL, Maceió, AL 
representativa de crianças e adolescentes, com idades de 7 a 17 anos, de ambos os gêneros, devidamente matriculados em escolas da rede de ensino público e particular, da zona urbana da cidade de Maceió, Alagoas.

As escolas foram selecionadas com base em informações oriundas da Unidade de Documentação e Informação (UDI) da Secretaria da Educação e do Desporto do Estado de Alagoas (Censo Escolar, 2000).

Para o cálculo do tamanho da amostra, levou-se em consideração a menor prevalência estimada de eventos estudados na linha de pesquisa sobre Fatores de risco cardiovascular em crianças e adolescentes da cidade de Maceió, da qual este estudo faz parte: hipertensão arterial sistêmica estimada em 7\% (III Consenso de Hipertensão Arterial da Sociedade Brasileira de cardiologia de 1998$)^{9}$, enquanto obesidade havia sido estimada em $10,2 \%^{10}$. Foi adotado um nível de confiança de 95\% e uma precisão, em torno da prevalência estimada, de dois pontos percentuais. O tamanho final da amostra foi de 1.253 estudantes.

A amostragem foi realizada por conglomerado e cada escola foi considerada um conglomerado. Foram sorteadas 40 escolas, correspondendo a aproximadamente $10 \%$ do número total de escolas (396 escolas) do município em estudo, assegurando uma adequada distribuição demográfica das escolas selecionadas na pesquisa. A seleção resultou na seguinte distribuição: 29 escolas públicas (01 federal, 20 estaduais e 08 municipais) e 11 escolas particulares.

Os alunos das escolas sorteadas foram selecionados por amostragem sistemática, por meio de lista ordenada do primeiro (primeiro da primeira turma) ao último (último da última turma), fornecida após consentimento da direção de cada escola (100\% das escolas sorteadas aceitaram).

No caso de recusa em participar da pesquisa $(0,3 \%)$ ou quando o aluno selecionado estava fora da faixa etária preconizada para o estudo (15,8\%), novo aluno era sorteado, utilizando a mesma sistemática. Obtivemos, previamente, consentimento livre e esclarecido dos pais e dos alunos sorteados para participarem da pesquisa.

O estudo foi aprovado pelo Comitê de Ética em Pesquisa do Hospital Universitário Prof. Alberto Antunes da Universidade Federal de Alagoas (UFAL).

Os dados foram coletados nas escolas por meio de questionário e medidas antropométricas que foram realizadas apenas pela pesquisadora do estudo, seguindo a padronização da OMS $(1995)^{11}$. A medida do peso corporal (Kg) foi obtida uma única vez, com os alunos descalços e vestindo short e camiseta como o mínimo de roupa possível, em balança digital (Indústrias Fillizola S.A. - Brasil) com capacidade de 0-150 Kg e precisão de 100 g devidamente aferida (INMETRO). Para a coleta da estatura foi utilizado um estadiômetro de madeira com escala metálica, capacidade de $2 \mathrm{~m}$ e precisão de 0,1 cm. A medida da estatura foi realizada em triplicata, utilizando-se o cálculo do valor médio. Os escolares foram mensurados descalços, em posição ereta, com calcanhares unidos e pés com as pontas afastadas, braços pendentes e com as mãos espalmadas sobre as coxas e em contato com a superfície plana do instrumento de medida. Cabeça ajustada ao plano de Frankfurt e em inspiração profunda. O Índice de Massa Corporal (IMC) foi obtido a partir da razão do peso corporal $(\mathrm{Kg})$ pelo quadrado da estatura $\left(\mathrm{m}^{2}\right)$.

O critério de referência para definir as variáveis relacionadas ao perfil nutricional foi a classificação do CDC (Centers for Disease and Control and Prevention) ${ }^{4}$, Atlanta, 2001, que é baseada no Índice de Massa Corporal (IMC): baixo peso (BP), IMC inferior ao percentil 5; peso normal (PN), IMC superior ao percentil 5 e inferior ao percentil 85; sobrepeso (SP), IMC superior ao percentil 85 e obesidade (OB), IMC igual ou superior ao percentil P95, para idade e sexo.

A análise estatística dos dados tabulados em planilha do programa Microsoft Excel foi realizada por meio da utilização do software SPSS (Statistical Package for Social Sciences), versão 13.0 para Windows. Para medir associação entre as variáveis, utilizou-se os testes do Qui quadrado $\left(X^{2}\right)$ e teste exato de Fisher. A análise de regressão logística foi utilizada para estimar a razão de chance (odds ratio /OR). As variáveis dependentes testadas foram sobrepeso e obesidade. As variáveis independentes foram gênero, idade e tipo de escola. Os resultados foram definidos como estatisticamente significantes para um valor de $p<0,05$ ou $5 \%$ para todos os testes.

\section{Resultados}

Foram avaliados 1253 alunos, sendo 547 do gênero masculino $(43,7 \%)$ e 706 do gênero feminino (56,3\%), com média de idade de $12,4 \pm 2,9$ anos. Considerando a escola que pertenciam, 21,3\% (267) foram das escolas particulares e 78,7\% (986) das escolas públicas.

Em relação à idade, observou-se que 19,9\% (249) dos alunos encontravam-se entre sete e nove anos, 52,7\% (660) entre 10 e 13 anos, e 27,4\% (344) entre 14 e 17 anos. Houve distribuição similar de ambos os gêneros, nas diversas faixas etárias e tipos de escola. As características da população estudada encontram-se na Tabela 1.

A prevalência total de sobrepeso e obesidade foi de $9,3 \%$ e $4,5 \%$, respectivamente, não havendo diferença estatisticamente significante entre gênero e sobrepeso $(p<0,58)$ e entre gênero e obesidade $(p<0,46)$.

A análise estatística bivariada demonstrou associação

\begin{tabular}{lc}
\hline \multicolumn{2}{c}{ Tabela 1 - Características da população estudada } \\
\hline Características & Número \\
\hline Idade (anos) & $12,4 \pm 2,92$ \\
Gênero & \\
Masculino & $547(43,7 \%)$ \\
Feminino & $706(56,3 \%)$ \\
Faixas etárias & \\
7 - 9 anos & $249(19,9 \%)$ \\
10 - 13 anos & $660(52,7 \%)$ \\
14 - 17 anos & $344(27,4 \%)$ \\
Escolas (alunos) & \\
Públicas & $986(78,7 \%)$ \\
Particulares & $267(21,3 \%)$ \\
Perfil Nutricional & \\
$<$ P5 & $83(6,6 \%)$ \\
P5 - P15 & $126(10 \%)$ \\
P15 - P85 & $872(69,6 \%)$ \\
P85 - P95 & $116(9,3 \%)$ \\
3 95 & $56(4,5 \%)$ \\
\hline
\end{tabular}


significante entre os alunos com sobrepeso e escola privada $(p<0,0001)$ e entre os alunos com obesidade e escola privada $(0,0001)$.

Quanto à obesidade, observou-se associação significante entre as seguintes faixas etárias: sete a nove anos $(p=0,04)$ quando comparada com 10 a 13 anos; e sete a nove anos quando comparada com 14 a 17 anos $(p=0,02)$.

A análise multivariada evidenciou que alunos de escola particular têm o dobro de chance de apresentar sobrepeso $(\mathrm{OR}=2,2$; IC95\%:1,36-3,32) e aproximadamente cinco vezes mais chances de apresentar obesidade (OR=4,7; IC95\%:2,32$9,34)$ em relação aos alunos da escola pública na amostra estudada.

\section{Discussão}

Segundo a $\mathrm{OMS}^{12}$, a obesidade é um problema de saúde frequente em países desenvolvidos e está rapidamente aumentando nos países em desenvolvimento, de forma que a prevalência da mesma em adultos é de $10 \%$ a $25 \%$ em países da Europa e de $20 \%$ a $25 \%$ em alguns países das Américas.

Em crianças e adolescentes, o seu aparecimento é preocupante por apresentar, mesmo nesta faixa etária, associação com alguns fatores de risco para as doenças cardiovasculares, bem como pela sua contribuição à morbimortalidade relacionada à obesidade no adulto ${ }^{13-15}$

Estudo de base populacional, representativo da população de Maceió (7 a 17 anos de idade), observou que a prevalência de pressão arterial elevada foi de 9,4\% e foi significativamente maior nos jovens com sobrepeso e obesidade, corroborando com os resultados encontrados nos estudos de associação de obesidade com demais de fatores de risco cardiovascular encontrados na literatura. ${ }^{16}$

Tornam-se imperativas estratégias de prevenção de âmbito populacional visando à promoção de estilos de vida saudáveis como dietas com baixa densidade energética, incremento na atividade física e mudanças de comportamento quanto ao controle do peso corporal $^{12}$. Tais estratégias são mais bem fundamentadas quando se reconhece a magnitude do problema e apresentam maior eficácia em crianças e adolescentes.

Este é o primeiro estudo epidemiológico, de base populacional, sobre o estado nutricional de escolares (crianças e adolescentes) realizado na cidade de Maceió. A ausência de outros estudos de base populacional no Brasil, na mesma faixa etária e utilizando os mesmos critérios diagnósticos impossibilitam a comparação dos resultados obtidos com outros de estudos similares.

A diversidade de métodos utilizados na caracterização do estado nutricional dos estudos relacionados a esta temática, além da etiologia multifatorial do sobrepeso e obesidade nessa faixa etária, torna a comparação entre os dados da literatura sujeitos a erros de interpretação.

A prevalência de $4,5 \%$ do sobrepeso na população total do presente estudo encontra-se abaixo da encontrada atualmente nos Estados Unidos, tomando-se como base aquela identificada a partir dos resultados iniciais do National Health and Nutrition Survey - (NHANES) de 199917, que é em média de 13\% para crianças de 6 a 11 anos e de 14\% para adolescentes de 12 a 19 anos. Na presente investigação, a prevalência do sobrepeso na população de 7 a 11 anos foi de $6,6 \%$ e na de 12 a 17 anos foi de $3,3 \%$. Estes resultados são próximos àqueles encontrados nos Estados Unidos entre 1976 e 1980, antes, portanto, das elevadas proporções ora observadas, o que pode sugerir que as crianças e adolescentes da cidade de Maceió apresentam ainda uma baixa prevalência de obesidade. Como o IMC no percentil igual ou acima de 95 tem sido aceito como um bom índice de excesso de adiposidade mesmo em crianças e adolescentes ${ }^{18-21}$, pode-se inferir, portanto, que a prevalência de obesidade na população estudada também foi baixa.

Utilizando os mesmos critérios adotados no presente estudo, pesquisadores identificaram uma prevalência de sobrepeso de 10,5\% em adolescentes de 11 a 17 anos, de ambos os gêneros, de uma escola pública do município de São Paulo (SP) ${ }^{22}$, sendo esta muito mais acentuada do que a verificada na presente investigação $(4,5 \%)$.

Pesquisadores identificaram obesidade em $8,5 \%$ de crianças e adolescentes, de 6 a 19 anos, de ambos os gêneros, em uma escola privada do Recife $(P E)^{8}$. Por meio dos mesmos critérios adotados nesse estudo, pesquisa em adolescentes de 15 a 17 anos, de ambos os sexos, de escola privada de Niterói (RJ), identificou uma prevalência de obesidade de 17,9\%8,23. Apesar de se tratar de amostras específicas (estudantes de escolas isoladas), não refletindo, portanto, o perfil dos municípios nos quais os estudos foram desenvolvidos, tais resultados ratificam a hipótese acima explicitada, de que a prevalência de sobrepeso e obesidade em crianças e adolescentes da cidade de Maceió é ainda muito baixa quando comparada à de países mais desenvolvidos que o Brasil ${ }^{17}$, com a de regiões mais desenvolvidas do Brasil.

Crianças e adolescentes que apresentam excesso de peso [sobrepeso + obesidade], IMC no percentil igual ou acima de 85, devem ser avaliados cuidadosamente para a identificação de excesso de gordura corporal e de complicações dela decorrentes ${ }^{18}$. Isto advém do fato de que nos mesmos o excesso de peso pode ser decorrente de aumento da gordura corporal (obesidade) ou da massa corporal isenta de gordura.

Enquanto no presente estudo a prevalência de sobrepeso foi de 9,3\%, nos estudos de Recife, São Paulo e Niterói a prevalência desta variável foi, respectivamente, de 26,2\%, 19,05\%, e 19,3\%8,21-22. Apesar da menor prevalência identificada nas crianças e adolescentes de Maceió, decorrente provavelmente dos mesmos fatores que explicam a menor prevalência de sobrepeso/obesidade na população estudada, a identificação de sobrepeso em 9,3\% dos estudantes estabelece a necessidade da avaliação clínica e acompanhamento dos mesmos, considerando que a manutenção dos componentes genéticos (imutáveis) e ambientais (passíveis de intervenção) que concorrem para a elevação progressiva do peso poderá determinar que os mesmos evoluam para a obesidade e seu amplo leque de comorbidades ${ }^{19-20,} 24$

$\mathrm{Na}$ presente investigação, o sobrepeso esteve significantemente associado à escola particular. A análise multivariada demonstrou que o estudante da escola particular tem duas vezes mais chances de apresentar sobrepeso do que o estudante da escola pública. Estudos indicam que apesar da baixa prevalência de obesidade observada em alguns países desenvolvidos, apenas as sociedades extremamente pobres parecem natural e consistentemente protegidas da mesma ${ }^{25}$. A elevação da prevalência 
da obesidade em países desenvolvidos e em desenvolvimento tem sido associada a dietas com excesso de gorduras e pobre em frutas e vegetais e à redução da atividade física no trabalho e no lazer (utilização do automóvel, horas em frente à televisão, ao computador, aos vídeo games) os quais se encontram indiscutivelmente associados ao poder aquisitivo dos indivíduos. À medida que melhora a condição econômica e os elementos acima citados se tornam acessíveis a todos, o processo de transição nutricional vai se estabelecendo.

\section{Conclusão}

De acordo com os resultados evidenciados no presente estudo, o sobrepeso e a obesidade em crianças e adolescentes ainda apresentam uma baixa prevalência quando associados à escola pública. Esta conclusão parece encontrar apoio no fato de que a obesidade também apresenta associação significante com a escola particular e a análise multivariada demonstra que o aluno da escola particular apresenta cinco vezes mais chances de ter obesidade do que o aluno da escola pública.

A obesidade foi significantemente mais frequente na faixa etária de sete a nove anos quando comparado às demais faixas etárias e tais resultados implicam na necessidade de acompanhamento longitudinal dos indivíduos com excesso de peso, considerando que o mesmo pode ser modificado ao longo do processo de crescimento, podendo, entretanto, ocorrer o risco da sua persistência e agravamento.

A ausência de dados anteriores, em Maceió, sobre o estado nutricional de indivíduos na faixa etária ora investigada, fundamenta a importância dos achados da presente investigação, considerando que além do diagnóstico atual, e sua inerente possibilidade de intervenção, a realização de estudos semeIhantes no futuro permitirá que sejam feitas análises de tendências quanto às prevalências de sobrepeso e obesidade.

Informações dessa natureza se constituem em ferramentas de inquestionável importância para as ações de saúde.

Os diferentes resultados encontrados, quando comparamos estudos relacionados a sobrepeso e obesidade em crianças e adolescentes, justificam-se pela influência ambiental de tais agravos, pela dinâmica cotidiana das regiões estudadas e pela diversidade de metodologias empregadas.

\section{AGRADECIMENTOS}

1- Os autores agradecem o auxílio (Processo 99/0268-7) da Fundação de Apoio à Pesquisa do Estado de Alagoas (FAPEAL) e ao Conselho Nacional de Desenvolvimento Científico e Tecnológico (CNPq) por meio do Programa Institucional de Bolsas de Iniciação Científica (PIBIC).

\section{Suporte Financeiro:}

Fundação de Amparo à Pesquisa do Estado de Alagoas (FAPEAL). Fonte Financiadora de Bolsas de Iniciação Científica: Programa de Bolsas de Iniciação Científica do CNPq/UFAL.

\section{Conflito de interesse: não há}

\section{SUMmARY}

Prevalence of oVerweight and obesity in CHILdRen AND ADOLESCENTS FROM THE CITY OF MACEIÓ (AL)

OBJECTIVE. To establish prevalence of overweight and obesity in children and adolescents from the city of Maceió, Alagoas, Brazil, and investigate the association of risk of overweight and obesity with gender, age and type of school.

Methods. This was a cross-sectional study. Students between 7 and 17 years of age were selected from 396 public and private schools of Maceió. After randomization, data were collected by questionnaire. Weight and height were measured (body mass index = weight:height2). Overweight and obesity were defined, respectively, as body mass index greater than the 85th percentile and equal or greater than the 95th percentile for age and gender.

RESULTS. The final sample included 1253 students (706 females). One hundred sixteen students were overweight and fifty six students were obese. Obesity was significantly associated with students between 7 and 9 years of age, when compared to the 10 and 13 year olds $(p<0.04)$ and the 14 and 17 year olds $(p<0.02)$. Private school students were more likely to be overweight (OR=2.2; $C 195 \%: 1.36$ - 3.32) and more likely to be obese (OR=4.7; C/95\%:2.32 - 9.34) than students from public schools.

CONCLUSION. The prevalence of overweight and obesity were, respectively, 9.3\% and 4.5\%. Overweight and obesity were significantly more frequent among private school students. [Rev Assoc Med Bras 2010; 56(2): 192-6]

KEY words: Prevalence. Overweight. Obesity. Child. Adolescent. Anthropometry.

\section{REFERÊNCIAS}

1. Troiano RP, Flegal KM, Kuczmarski RJ, Campbell SM, Johnson CL. Overweight prevalence and trends for children and adolescents. The National Health and Nutrition Examination Surveys, 1963 to 1991. Arch Pediatr Adolesc Med. 1995; 149:1085-91.

2. Monteiro CA, Conde WL. A tendência secular da obesidade segundo estratos sociais: Nordeste e Sudeste do Brasil, 1975-1989-1997. Arq Bras Endocrinol Metab. 1999;43:186-94.

3. Popkin BM, Doak C. The obesity epidemic is a worldwide phenomenon. Nutr Rev. 1998;56:106-14.

4. CDC (Center for Disease Control and Prevention [homepage on the Internet]. Body Mass Index-for-age- BMI is used differently with children than it is with adults. Atlanta: CDC; 2002. [cited 2009 jan 16]. Available from: http://www. cdc.gov/nccdphp/dnpa/bmi/bmi-for-age.htm.

5. Duran-Tauleria E, Rona JR, Chinn S. Factors associated with weight for height and skinfold thickness in British children. J Epidemiol Commun Health. 1995; 49:466-473

6. Engestrom EM, Anjos LA. Relação entre o estado nutricional materno e sobrepeso nas crianças brasileiras. Rev Saúde Pública. 1996;30:233-9.

7. Monteiro CA, Mondini L, Medeiros de Souza AL, Popkin BM. The nutrition transition in Brazil. Eur J Clin Nutr. 1995;49:105-13.

8. Balaban G, Silva GAP. Prevalência de sobrepeso e obesidade em crianças e adolescentes de uma escola da rede privada do Recife. J Pediatria. 2001;77:96-100.

9. Sociedade Brasileira de Cardiologia. SBC - Sociedade Brasileira de Cardiologia 1996-2002. III Consenso Brasileiro de Hipertensão Arterial, 1998. [citado 16 jan 2009]. Disponível em: http://departamentos.cardiol.br/dha/publicacoes/ consenco3/consen.asp.

10. Mondini D, Monteiro CA. Relevância epidemiológica da desnutrição e da obesidade em distintas classes sociais: métodos de estudo e aplicação a população brasileira. Rev Bras Epidemiol. 1998;1:28-39.

11. World Health Organization. Physical status: the use and interpretation of anthropometry. Report of a WHO Expert Committee. Geneva: WHO: 1995.

12. World Health Organization. WHO - World Health Organization - 2002. What is cardiovascular disease? [cited 2009 apr 12]. Available from: http://www. who.int/ncd/cvd/index.htm.

13. Williams DP, Going SB, Lohman TG, Harsha DW, Srinivasan SR, Weber IS. Body fatness and risk for elevated blood pressure, total cholesterol and serum lipoprotein ratios in children and adolescents. Am J Public Health. 1992;82:358-63.

14. Raitakari OT, Porka KVK, Rõnemaa T, Akerblom HK. Clustering of risk factors for coronary heart disease in children and adolescents: the Cardiovascular Risk in Young Finns Study. Acta Paediatr Jpn. 1994;83:935-40.

15. Dietz WH. Health consequences of obesity in youth: childhood predictors of adult disease. Pediatrics. 1998;101:518-25. 
16. Moura AA, Silva MAM, Ferraz MRMT, Rivera IR. Prevalência de pressão arterial elevada em escolares e adolescentes de Maceió. J Pediatria. 2004;80(1):35-40.

17. National Center for Health Statistics. NCHS - National Center for Health Statistics - 2002. Prevalence of overweight among children and adolescents: United States. Updated 1999. [cited 2009 Feb 17]. Available from: http://www.cdc.gov/nchs/products/pubs/pubd/hestats/overwght99.htm.

18. Must A, Dallal G, Dietz WH. Reference data for obesity: $85^{\text {th }}$ and $95^{\text {th }}$ percentiles of body mass index and triceps skinfold thickness. Am J Clin Nutr. $1991 ; 53: 839-46$

19. Himes JH, Dietz WH. Guidelines for overweight in adolescent preventive services: recommendations from an expert committee. Am J Clin Nutr. 1994;59:307-16

20. Barlow SE, Dietz WH. Obesity evaluation and treatment: Expert Committee Recommendations. Pediatrics. 1998;102(3):e29.

21. Dietz WH, Bellizzi MC. Introduction: the use of body mass index to assess obesity in children. Am J Clin Nutr. 1999;70(suppl):123S-25S.
22. Albano RD, Souza ZA. Estado nutricional de adolescentes: risco de sobrepeso e sobrepeso em uma escola pública do Município de São Paulo. Cad Saúde Pública. 2001;17:941-47.

23. Fonseca VM, Sichieri R, Veiga GV. Fatores associados à obesidade em adolescentes. Rev Saúde Pública. 1998;32:541-49.

24. Moran R. Evaluation and treatment of childhood obesity. Am Fam Physician. 1999;59:861-8,871-3.

25. Monteiro CA. Epidemiologia da obesidade In: Halper A, Matos AFG, Suplicy HL, Mancini MC, Zanella MT, editores. Obesidade. São Paulo: Lemos Editorial; 1998. p.15-30.

Artigo recebido: 05/11/09

Aceito para publicação: 20/01/10 